\title{
Hepatorenal Syndrome in the Emergency Department: A Case Report
}

Win Jim Tan, ${ }^{1}$ Mohan Tiruchittampalam. ${ }^{2}$

\begin{abstract}
Background: Hepatorenal syndrome is a condition where there is functional renal failure in a background of liver disease. It is relatively common in patients with liver cirrhosis and is associated with a high mortality rate if untreated. Case: This is a case report of an 88-year-old Chinese man presenting from a community hospital with a new onset of abdominal distension on a background of cryptogenic liver cirrhosis diagnosed on computed tomography scan. Clinical history and physical findings were consistent with that of fluid overload. Investigations performed indicated acute kidney injury together with liver failure secondary to liver cirrhosis. The patient was diagnosed with hepatorenal syndrome in accordance with the criteria established by the International Ascites Club and managed with an infusion of vasopressin and albumin in the emergency department. He was subsequently admitted to the general ward (gastrology), where he was managed for hepatorenal syndrome, improved clinically and was discharged to the nursing home. Conclusion: Hepatorenal syndrome can be managed effectively with albumin and vasopressin, and such treatment can be started as early as in the emergency department. Acute care physicians should not be hesitant in diagnosing and treating hepatorenal syndrome as early as in the emergency department for appropriate patients.
\end{abstract}

Keywords: Hepatorenal Syndrome, Liver Cirrhosis, Albumins, Vasopressins, Vasopressor Agents (Source: MeSH, NLM).

About the Author: Win Jim Tan is a final-year medical student in the 5-year MBBS program at Yong Loo Lin School of Medicine, National University of Singapore.
Submission: Jul 27, 2014

Acceptance: Nov 8, 2014

Publication: Dec 31, 2014

Process: Peer-reviewed

\section{Introduction}

Hepatorenal syndrome (HRS) is a potentially reversible condition where there is functional renal failure in a background of liver disease. It is relatively common, with an incidence of 1 in 10 hospitalized patients with liver cirrhosis in the United States of America. ${ }^{1}$ This is of great concern given the high mortality rate associated with HRS if left untreated. Type 1 HRS is associated with rapid progression to renal failure over as short a period as two weeks, compared to Type 2 HRS which is associated with a slow but steady progressive course with refractory ascites. Median survival time in patients with untreated type 1 HRS is two to four weeks, with $95 \%$ of such patients dying within the first 30 days of onset. Those with type 2 HRS have a median survival time of 6 months. ${ }^{2}$ The International Ascites Club (IAC) has provided a set of criteria revised in 2005 to aid in the diagnosis of HRS (Table 1 ).

Given the high mortality rate associated with untreated HRS and the potential to reverse this syndrome, it is of utmost importance for clinicians to identify patients with HRS as per the IAC criteria and initiate appropriate treatment as soon as possible. ${ }^{2}$ This report concerns a patient presenting to the emergency department of a tertiary hospital with HRS. This report serves to highlight the role acute care practitioners can play in diagnosing HRS and starting treatment early. The case reports statement (CARE) framework was followed in the drafting of this case report.

\section{The Case}

An 88-year-old Chinese man was referred from a community hospital when the caregiver noted abdominal distension

\section{Key Points:}

- Hepatorenal syndrome is a common complication of liver cirrhosis. - If left untreated, hepatorenal syndrome, particularly type 1 with spon taneous bacterial peritonitis, is associated with a high mortality rate. - Albumin and vasopressors have been proven to be a good treatment option for hepatorenal syndrome.

- Early institution of treatment is important in managing hepatorenal syndrome, and treatment may be instituted as early as in the emergency department.

Table 1. The International Club of Ascites Criteria for the Diagnosis of Hepatorenal Syndrome.

\section{Presence of cirrhosis and ascites 2. Serum creatinine $>1.5 \mathrm{mg} / \mathrm{dL}$ (or $133 \mu \mathrm{mol} / \mathrm{L}$ ) \\ 3. No improvement of serum creatinine (decrease equal to or less than $1.5 \mathrm{mg} / \mathrm{dL}$ ) after at least 48 hours of diuretic withdrawal and volume expansion with albumin (recommended dose: $1 \mathrm{~g} / \mathrm{kg}$ b.w. per day up to a maximum of $100 \mathrm{~g}$ of albumin/day)}

\section{Absence of shock}

5. No current or recent treatment with nephrotoxic drugs

6. Absence of parenchymal kidney disease as indicated by proteinuria $>500 \mathrm{mg} /$ day, microhematuria ( $>50$ red blood cells/high power field, and/ or abnormal renal ultrasound scanning

(Available from: http://www.icascites.org/about/guidelines/, updated 2005 Nov; cited 2014 Nov 16).

while aiding the patient in rehabilitation. The patient had a past medical history of type 2 diabetes mellitus, hypertension, paroxysmal atrial fibrillation (PAF) and sick sinus syndrome for which he had a VVI pacemaker inserted, right hemispheric syndrome secondary to right middle cerebral artery infarction complicated by hemorrhagic conversion, and a background of

${ }^{1}$ National University of Singapore, Singapore.

${ }^{2}$ Changi General Hospital, Singapore. 
cryptogenic liver cirrhosis corroborated with computed tomography (CT) findings which showed established liver cirrhosis and no evidence of obstructive nephropathy or parenchymal disease. He was on gliclazide and metformin for diabetes control, atorvastatin for dyslipidemia, amlodipine, enalapril and bisoprolol for hypertension, and aspirin for PAF. There was no recent change in medications.

On physical examination, the patient had anasarca, with a body temperature of $37.2^{\circ} \mathrm{C}$, pulse rate of 60 beats per minute, respiratory rate of 16 breaths per minute, blood pressure of $132 / 72 \mathrm{mmHg}$, and oxygen saturation of $100 \%$ on room air. The patient was drowsy, groaned and localized pain. His abdomen was soft and distended, with a positive fluid thrill and shifting dullness. Pitting edema was noted in bilateral lower limbs. Cardiovascular and respiratory examination yielded no significant findings, and the lungs were clear on auscultation. The patient was managed in the resuscitation area.

Hematological investigations were performed. Liver panel showed transaminitis with elevated alanine transaminase (ALT) at $78 \mathrm{U} / \mathrm{L}$ and aspartate transaminase (AST) at $77 \mathrm{U} / \mathrm{L}$ (Table 2). Renal panel showed markedly elevated urea at $37.8 \mathrm{mmol} / \mathrm{L}$ and mildly elevated creatinine at $133.0 \mu \mathrm{mol} / \mathrm{L}$ at presentation, rising from a baseline of $12.9 \mathrm{mmol} / \mathrm{L}$ for urea and $83.0 \mu \mathrm{mol} / \mathrm{L}$ for creatinine at one month prior to presentation. Mild hyperkalemia was also noted with a serum potassium level of 5.9 $\mathrm{mmol} / \mathrm{L}$. Other hematological investigations had values within normal ranges. Initial urinalysis showed no evidence suggestive of a parenchymal kidney disease, with the absence of proteinuria and microscopic hematuria. Analysis of the ascitic fluid ruled out occult sources of infection in this patient.

Differential diagnoses considered included fluid overload secondary to acute renal failure and deterioration of clinical condition secondary to underlying liver cirrhosis. The diagnosis of HRS could not be excluded based on the patient's clinical presentation. The patient had a background of cirrhosis and ascites and was not on any nephrotoxic drugs. He was not clinically in shock and was hemodynamically stable. Serum creatinine was elevated at $133.0 \mu \mathrm{mol} / \mathrm{L}$ with no evidence of parenchymal kidney disease. Considering the above clinical and laboratory findings together with the presentation of renal failure in a background of liver cirrhosis, the patient was provisionally diagnosed with HRS as per the IAC criteria (Table 1). With the prompt diagnosis, he was started on intravenous (IV) infusion of vasopressin at a rate of $0.01 \mathrm{U} / \mathrm{min}$ and albumin $5 \%$ in $500 \mathrm{ml}$ over two hours. IV insulin at $5 \mathrm{U} / \mathrm{h}$ and IV dextrose $5 \%$ in $500 \mathrm{ml}$ over eight hours were also started for the management of hyperglycemia. A nasogastric tube was also inserted for enteral nutrition given a previous history of dysphagia secondary to right middle cerebral artery infarction with hemorrhagic conversion.

In view of the diagnosis, the patient was admitted to the general medicine ward (gastrology). The patient's diagnosis of HRS was confirmed in the ward setting, and he was managed accordingly. He subsequently improved clinically and was discharged to a nursing home.

\section{Discussion}

This case report demonstrated that HRS can be diagnosed early and managed accordingly even in the acute care setting. ${ }^{3}$ Diagnosis of HRS can be achieved with the aid of the criteria developed by IAC, and appropriate treatment can be started even before the patient is admitted to the ward.

The pathogenesis of HRS is currently still being explored. Arterial vasodilatation of the splanchnic circulation associated with the local release of vasodilatory substances is considered as a possible explanation. Renal vasoconstriction in the absence of reduced cardiac output and blood volume may also contribute to the development of HRS. ${ }^{4} \mathrm{~A}$ high index of suspicion for HRS should be present in all cases with liver cirrhosis presenting with non-specific symptoms such as malaise and an acute decline in renal function. ${ }^{2}$ It must also be noted that patients with type 1 HRS associated with spontaneous bacterial peritonitis have almost $100 \%$ hospital mortality rate if appropriate treatment is not provided. ${ }^{5}$

Given the wide variety of causes which may be associated with renal failure in patients with underlying cirrhosis, the IAC deve-

Table 2. Relevant Findings of Renal and Liver Function Tests and Urinalysis at Presentation and One Month Prior to Presentation.

\begin{tabular}{|c|c|c|c|}
\hline Test & Reference Range & Feb 2014 & Mar 2014 \\
\hline Urea, serum $(\mathrm{mmol} / \mathrm{L})$ & $2.8-7.7$ & 12.9 & 37.8 \\
\hline Sodium, serum (mmol/L) & $135.0-145.0$ & 135.0 & 146.0 \\
\hline Potassium, serum $(\mathrm{mmol} / \mathrm{L})$ & $3.5-5.3$ & 4.5 & 5.9 \\
\hline Chloride, serum (mmol/L) & $96.0-108.0$ & 109.0 & 119.0 \\
\hline Bicarbonate, serum (mmol/L) & $19.0-31.0$ & 17.0 & 15.0 \\
\hline Glucose, serum (mmol/L) & $3.1-7.8$ & 7.2 & 16.5 \\
\hline Creatinine, serum $(\mu \mathrm{mol} / \mathrm{L})$ & $50-90$ & 83 & 133 \\
\hline Albumin, serum (g/L) & $37-51$ & $\mathrm{~N} / \mathrm{A}$ & 24 \\
\hline Alanine transaminase, serum $(\mathrm{U} / \mathrm{L})$ & $10-55$ & $\mathrm{~N} / \mathrm{A}$ & 78 \\
\hline Aspartate transaminase, serum $(\mathrm{U} / \mathrm{L})$ & $10-45$ & $\mathrm{~N} / \mathrm{A}$ & 77 \\
\hline Red blood cells, urine (cells/high power field) & $0-3$ & $\mathrm{~N} / \mathrm{A}$ & 0 \\
\hline White blood cells, urine (cells/high power field) & $0-6$ & $\mathrm{~N} / \mathrm{A}$ & 13 \\
\hline Epithelial cells, urine (cells/high power field) & $0-4$ & $\mathrm{~N} / \mathrm{A}$ & 0 \\
\hline Protein, urine & Negative & $\mathrm{N} / \mathrm{A}$ & Negative \\
\hline
\end{tabular}




\section{Case Report}

loped a revised set of criteria in 2005 to aid in the diagnosis of HRS. ${ }^{3}$ The utility of such criteria cannot be understated. With a $10 \%$ incidence rate among hospitalized patients and with the high morbidity and mortality associated with untreated HRS, early diagnosis and institution of appropriate treatment is of definite importance in these patients..$^{1,2,6}$

The mainstay of therapy for patients with HRS remains liver transplantation. Published literature has shown the effectiveness of transplantation in ensuring survival and good renal function in patients with HRS.? It must however be noted that liver transplantation may not be suitable for all HRS patients. The unsuitability for liver transplantation, coupled with the long waiting times at most transplant centers, demands the search for alternative therapies for HRS.

Several pharmacological options are available in the management of HRS. While vasodilators such as dopamine were initially thought to be effective, the current practice has shifted to the use of vasoconstrictors. Several studies have shown that vasoconstrictors acting on the splanchnic circulation, including vasopressin and its analogues terlipressin and ornipressin, are able to improve patient outcome. It is postulated that these vasoconstrictors have an effect on the splanchnic circulation, reversing the vasodilatory effect usually found in HRS. ${ }^{8}$ Despite a trial showing that terlipressin is more effective than norepinephrine in treating HRS, the utility of norepinephrine and other similar drugs as an alternative must not be discounted. ${ }^{9}$

The use of albumin together with vasopressors has also been shown to provide a good outcome, particularly in patients diagnosed with type 1 HRS associated with spontaneous bacterial peritonitis. ${ }^{5}$ The IAC has recommended the use of albumin as the preferred volume expander in patients with HRS. ${ }^{3}$ For this reason, a combination therapy involving both albumin and a suitable vasopressor such as terlipressin should be considered when initiating pharmacological management of HRS.
The strengths of this case report include the fact that there is little literature regarding prompt diagnosis and treatment of HRS, particularly in the acute care setting. We demonstrated that HRS can be diagnosed promptly using the criteria developed by IAC and managed accordingly even in the acute care setting. As the corroborative literature mentioned in the discussion has shown, early measures to tackle HRS are important in providing a better outcome for these patients.

We acknowledge the limitations of our case report. Given that this patient was managed in the acute care setting, the luxury of time was not afforded to us in our management of the case. Hence, the primary priority in this case was to provide acute care for the patient and to stabilize him for further treatment by the gastrologist in the ward setting. In this instance, other investigations such as ascitic fluid analysis to rule out spontaneous bacterial peritonitis could only be performed after the patient was transferred to the ward.

\section{Conclusion}

Several learning points can be obtained from this case report. From the above discussion regarding medical literature concerning HRS, early diagnosis and initiation of appropriate treatment is definitely important in managing HRS. Acute care clinicians should consider HRS as a possible diagnosis in patients presenting with a new onset of renal failure and with a background of liver cirrhosis. Albumin and vasopressors can and should be started as early as in the emergency department in patients who are suspected of having HRS. Acute care physicians should not be hesitant in diagnosing and starting the treatment of HRS in the appropriate patients particularly in the emergency department. Further studies to be considered include investigating the benefit of starting HRS treatment in the emergency department as compared to the ward setting. 


\section{References}

1. Carvalho GC, Regis Cde A, Kalil JR, Cerqueira LA, Barbosa DS, Motta MP, et al. Causes of renal failure in patients with decompensated cirrhosis and its impact in hospital mortality. Ann Hepatol. 2012 Jan-Feb;11(1):90-5.

2. Gines P, Guevara M, Arroyo V, Rodes J. Hepatorenal syndrome. Lancet. 2003 Nov 29;362(9398):1819-27.

3. Salerno F, Gerbes A, Gines P, Wong F, Arroyo V. Diagnosis, prevention and treatment of hepatorenal syndrome in cirrhosis. Gut. 2007 Sep;56(9):1310-8. 4. Arroyo V, Terra C, Gines P. Advances in the pathogenesis and treatment of type-1 and type-2 hepatorenal syndrome. J Hepatol. 2007 May;46(5):935-46. 5. Sort P, Navasa M, Arroyo V, Aldeguer X, Planas R, Ruiz-del-Arbol L, et al. Effect of intravenous albumin on renal impairment and mortality in patients with cirrhosis and spontaneous bacterial peritonitis. N Engl J Med. 1999 Aug 5;341(6):403-9.
6. Gines A, Escorsell A, Gines P, Salo J, Jimenez W, Inglada L, et al. Incidence, predictive factors, and prognosis of the hepatorenal syndrome in cirrhosis with ascites. Gastroenterology. 1993 Jul; 105(1):229-36.

7. Gonwa TA, Morris CA, Goldstein RM, Husberg BS, Klintmalm GB. Long-term survival and renal function following liver transplantation in patients with and without hepatorenal syndrome--experience in 300 patients. Transplantation. $1991 \mathrm{Feb}$;51(2):428-30.

8. Treschan TA, Peters J. The vasopressin system: physiology and clinical strategies. Anesthesiology. 2006 Sep;105(3):599-612;quiz 639-40.

9. Alessandria C, Ottobrelli A, Debernardi-Venon W, Todros L, Cerenzia MT, Martini $S$, et al. Noradrenalin vs terlipressin in patients with hepatorenal syndrome: a prospective, randomized, unblinded, pilot study. J Hepatol. 2007 oct; $47(4): 499-505$

\section{Acknowledgments}

None.

Conflict of Interest Statement ct Funding

The author has no funding, financial relationships or conflicts of interest to disclose.

Author Contributions

Conception and design the work/idea: WJT. Collect data/obtaining results: WJT. Write the manuscript: WJT. Critical revision of the manuscript: MT. Approval of the final version: MT. Administrative or technical advice: MT.

Cite as:

Tan WJ, Tiruchittampalam M. Hepatorenal Syndrome in the Emergency Department: A Case Report. Int J Med Students. 2014 Nov2015 Mar;3(1):51-4. 